By this means I made comparisons on the mornings of the $9^{\text {th }}$ and $10^{\text {th }}$ with the star B.A.C. 3303 . The transits were observed on opposite sides of the square, and are,

therefore, not so satisfactory as I could wish. The following are the results corrected for differential refraction and the comets proper motion.

\begin{tabular}{|c|c|c|c|c|c|c|c|c|}
\hline r 882 & Winds. M. T. & $\begin{array}{c}\text { Comet } \\
\text { Diff. } \\
\text { App. R.A. }\end{array}$ & $\begin{array}{c}\text {-Star } \\
\text { Diff. } \\
\text { App. N.P.D. }\end{array}$ & Comp. & $\begin{array}{r}\text { Comet's } \\
\text { App. R. A. }\end{array}$ & $\log \frac{p}{P}$ & $\begin{array}{c}\text { Comet's } \\
\text { App. N.P.D. }\end{array}$ & $\log \frac{q}{p}$ \\
\hline Sept. 8 & I $7^{\mathrm{h}} 54^{\mathrm{m}} 5^{2^{\mathrm{s}}}$ & $+3^{\mathrm{m}} 15^{\mathrm{s}} \cdot 90$ & $+2 I^{\prime} 8.0$ & 4 & $9^{\mathrm{h}} 37^{\mathrm{m}} \quad 7^{\mathrm{s}} \cdot 5 \mathrm{o}$ & $8.7 \times 15 \mathrm{n}$ & $90^{\circ} 57^{\prime} 46^{\prime \prime} \cdot 4$ & $9.73^{65}$ \\
\hline 9 & $\begin{array}{lll}17 & 49 & 45\end{array}$ & +1156.19 & +1657.9 & 2 & $\begin{array}{lll}9 & 45 & 47.8 \mathrm{I}\end{array}$ & $8.7 \times 87 n$ & $\begin{array}{lll}90 & 53 & 36.2\end{array}$ & 9.7370 \\
\hline
\end{tabular}

The star's mean place for 1882.0 and the reductions to the dates of observation are as follow:

$\begin{array}{cccc}\text { Mean R.A. } & \text { Reduction } & \text { Mean N.P.D. } & \text { Reduction } \\ 9^{\mathrm{h}} 33^{\mathrm{m}} 49^{\mathrm{s}} .76 & +1.8 \dot{4} & 90^{\circ} 36^{\prime} 27^{\prime \prime} 6 & +10^{\prime \prime} 8 \\ & +1.86 & \$ & +10.7\end{array}$

The authorities for the star's place are Schjellerup, Radcliffe Obs. I 858 and Annales de l'Obs. Royal de Bruxelles, Cat. I874. Up to the present date I have not succeeded in getting further observations owing to fog and cloud, but it is probable that the comet will soon become visible in the West.

Windsor N. S. Wales, I 882 September 16 .

Fohn Tebbutt.

\title{
Ueber eine dreifache Lösung des Cometenproblems.
}

In meinem demnächst erscheinenden Lehrbuch zur Bahnbestimmung der Cometen und Planeten (2. Auflage) trete ich in einem Kapitel, welches uiber die mehrfachen Lösungen des Cometenproblems handelt, der allgemein getheilten Ansicht entgegen, dass das Cometenproblem in Folge des doppelten Zeichens einer auftretenden Wurzel eine doppelte Lösung zulasse; ich beweise, dass nur das. positive Zeichen Geltung habe, und weiter, dass für die geocentrischen Distanzen entweder nur eine oder drei positive Lösungen stattfinden können. Als ich gestern Nr. 2464 der Astr. Nachr. mit der hochinteressanten Mittheilumg des
Dr. H. Oppenheim über eine doppelte Lösung bei der Bahnberechnung des Cometen Cruls aus den drei Coimbrabeobachtungen erhielt, war es mir sofort klar, dass noch eine dritte brauchbare Wurzel der Gleichung vorhanden sein müsse, und ich habe diese Entscheidung sofort brieflich Dr. Oppenheim mitgetheilt. Um aber auch von meiner Seite das Vorhandensein der dritten Wurzel durch Rechnung nachzuweisen, habe ich die hierfür nothwendigen kleinen Rechnungen durchgeführt; die Bahnbestimmung gründet sich auf die folgenden Daten (mittl. Aeq. I 882.0):

\begin{tabular}{|c|c|c|c|c|}
\hline Mittl. Berliner Zeit & $\lambda$ & $\beta$ & $L$ & $\log R$ \\
\hline I 88 z Sept. I 8.04782 & I $72^{\circ} 46^{\prime} 57^{\prime \prime} 6$ & $-1^{\circ} 37^{\prime} \times 4^{\prime \prime} 6$ & I $75^{\circ} 27^{\prime} \quad 4^{\prime \prime} 9$ & 0.001827 \\
\hline 19.03886 & I 7 I 547.9 & -32326.1 & I $76 \quad 25 \quad 12.7$ & $0.00 \times 706$ \\
\hline 20.03157 & I $69 \quad 5429 . I$ & $\begin{array}{lll}-4 & 48 & 20.3\end{array}$ & I 772328.1 & 0.001583 \\
\hline
\end{tabular}

Die Auflösung der bekannten Gleichungen führt in der That auf die folgenden 3 Lösungen ( $\rho_{1}$ und $\varrho_{3}$ die geocentrischen Distanzen):

\begin{tabular}{|c|c|c|c|}
\hline & Lösung & II. Lösung & III. Lösung \\
\hline & $9 \cdot 548488$ & 9.869946 & 0.032510 \\
\hline $\begin{array}{l}\log Q \\
\log r\end{array}$ & $\begin{array}{l}9.57 \times 291 \\
9.813879\end{array}$ & $\begin{array}{l}9.892749 \\
9.42678 \mathrm{I}\end{array}$ & $\begin{array}{l}0.0553 \text { I } 3 \\
8.967676\end{array}$ \\
\hline $\log r$ & 9.804878 & $9.4 \times 7259$ & 9.325809 \\
\hline
\end{tabular}

die zu den folgenden Elementen führen:

$$
\begin{aligned}
& T 1882 \text { Sept. } 28.18658 \quad \text { I } 9.72362 \quad \text { I 7.1 } 2868 \\
& \pi \quad \ldots \ldots \ldots 25^{\circ} 20^{\prime} 4 I^{\prime \prime} \cdot 5 \quad 2 I^{\circ} 2 I^{\prime} \quad 3 I^{\prime \prime} \cdot 0 \quad 64^{\circ} 4^{\prime \prime} \quad 5^{\prime \prime} 5 \\
& \begin{array}{lllllllllllll}
8 \ldots \ldots & \ldots & 44 & 41 & 42.4 & 175 & 18 & 34.8 & 347 & 57 & 48.3
\end{array}
\end{aligned}
$$

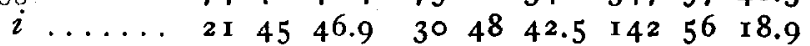

$$
\begin{aligned}
& \log 9 \ldots \ldots \quad 9.787 \times 60 \quad 9.416916 \quad 8.110866 \text {. }
\end{aligned}
$$

An der oben erwähnten Stelle bemerke ich noch, dass man durch die Darstellung der mittleren Beobachtung meist wird zur Entscheidung bringen können, welche Wurzel die wahre sei. In der That finden sich durch diese drei Elemente in der mittleren Beobachtung die folgenden Fehler: 


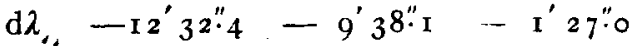

$$
\begin{aligned}
& \mathrm{d} \beta_{\prime \prime \prime}^{\prime \prime}-5^{6.6}-66^{6}-\circ 59.1
\end{aligned}
$$

Rechnet man in der bekannten Weise den Logarithmus der Cotangente des Winkels, den der durch die mittleren Cometen- und Sonnenorte gelegte grösste Kreis mit der Ekliptik einschliesst, so findet sich mit Benutzung

$\begin{array}{ccc}\text { der Beobachtungen } \log \operatorname{cotg} \mathcal{F} & 0.194795 \\ \text { mit dem ersten Elementensystem } & 0.194781 \\ \# \# \text { zweiten } & 0.19479^{2} \\ » \# \text { dritten } & & 0.194937\end{array}$

Es zeigt sich hieraus, dass durch eine Verbesserung in $M$ für die beiden ersten Lösungen kein wesentlich besserer Anschluss an die Beobachtungen erreicht werden kann, während sich $M$ für das dritte System verbesserungsfähig erweist; es ist demnach gar keinem Zweifel unterworfen, wie dies wohl schon anderweitig bekannt ist, dass die dritte Lösung die einzig brauchbare ist.

Wien 1882 October 29.

Th. v. Oppolzer.

\section{Ueber die Kriterien des Vorhandenseins dreier Lösungen bei dem Cometenproblem.}

In einer der kais. Akademie der Wissenschaften in Wien von mir überreichten Abhandlung führen sich die Kriterien für das Vorhandensein dreier Lösungen bei dem Cometenproblem auf die unten folgenden Formeln zurück.

Sind $h, g$ und $\cos \varphi$ die bekannten bei einer Cometenbahnbestimmung auftretenden Hilfsgrössen, $\lambda_{\mu}$ und $\beta_{u}$ die

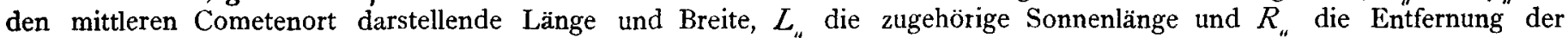
Erde von der Sonne, so ist'zunächst zu berechnen

und weiter:

$$
\alpha=\frac{h}{g} R_{u}, \cos \psi_{u}=\cos \beta_{n} \cos \left(\lambda_{n}-L_{u}\right)
$$

$$
\begin{aligned}
A_{1} & =-\frac{5}{3} \cos \psi_{\circ}-\frac{4}{3}\left(\frac{\cos \varphi}{\alpha}\right), & A_{2} & =\frac{2}{3}+2 \cos \psi_{1}\left(\frac{\cos \varphi}{\alpha}\right)+\frac{1}{3 \alpha^{2}} \\
A_{3} & =-\frac{2}{3}\left(\frac{\cos \varphi}{\alpha}\right)-\frac{\cos \psi_{1}}{3 \alpha^{2}}, & p & =\frac{\mathrm{I}}{3} A_{1}{ }^{2}-A_{2} \\
q & =\frac{2}{27} A_{1}{ }^{3}-\frac{\mathrm{I}}{3} A_{1} A_{2}+A_{3}, & r^{2} & =\frac{4}{3} p, \sin 3 \omega=\frac{4 q}{r^{3}},
\end{aligned}
$$

$p$ muss positiv und $4 q<r^{3}$ sein, wenn überhaupt drei brauchbare Lösungen vorhanden sein sollen; $r$ stets positiv und 300 im ersten Quadranten zu wählen und 300 erhält das Vorzeichen von $q$;

$$
x_{2}=r \sin \omega-\frac{\mathrm{I}}{3} A_{1}, x_{3}=r \sin \left(60^{\circ}-\infty\right)-\frac{\mathrm{I}}{3} A_{1}
$$

$x_{2}$ muss positiv sein, wenn überhaupt drei brauchbare Lösungen möglich sein sollen; dieselben sind in der That vorhanden, wenn den beiden Ungleichungen:

genügt .wird.

$$
\begin{aligned}
& \left(\alpha^{2} x_{2}{ }^{2}-2 \cos \varphi \cdot \alpha x_{2}+1\right)^{2}\left(x_{2}{ }^{2}-2 \cos \psi_{u} \cdot x_{2}+1\right)>4 R^{2}{ }^{2} \\
& \left(\alpha^{2} x_{3}{ }^{2}-2 \cos \varphi \cdot \alpha x_{3}+1\right)^{2}\left(x_{3}{ }^{2}-2 \cos \psi_{1} \cdot x_{3}+1\right)<4 \mathrm{R}{ }^{2}{ }^{2}
\end{aligned}
$$

Für das in der letzen Zeit am Cometen Cruls entdeckte Beispiel wird:

$$
\begin{aligned}
& \log \alpha=0.4427, \log \cos \psi_{u}=9.9974, \log \cos \varphi=9.7793 \mathrm{n}, \log \mathrm{R}_{u}=0.00 \mathrm{I} 7 \\
& A_{1}=-\mathrm{r.3674}, A_{2}=+0.2786, \quad A_{3}=+0.1016, \quad p=+0.3447 \\
& q=+0.0393, \log r=0.8312, \quad \omega=10^{\circ} 6^{\prime}, \quad x_{2}=+0.5747, x_{3}=+0.9743 \\
& \left(\alpha^{2} x_{2}^{2}-2 \cos \varphi \cdot \alpha x_{2}+1\right)^{2}\left(x_{2}{ }^{2}-2 \cos \psi_{11} \cdot x_{2}+1\right)=+5 \cdot 6 \\
& 4 R^{2}=+4.0 \\
& \left(\alpha^{2} x_{3}{ }^{2}-2 \cos \varphi \cdot \alpha x_{3}+1\right)^{2}\left(x_{3}{ }^{2}-2 \cos \psi_{10} \cdot x_{3}+1\right)=+ \text { I. }{ }^{4} \text {. }
\end{aligned}
$$

Wien 1882 November 3 .

Th. v. Oppolzer, 